\title{
Automatic artery/vein classification in colour retinal images
}

Martinez-Perez, M. Elena, Parker, Kim, Witt, Nick, Hughes, Alun, Thom, Simon A.

M. Elena Martinez-Perez, Kim H. Parker, Nick Witt, Alun D. Hughes, Simon A. $M$. Thom, "Automatic artery/vein classification in colour retinal images," Proc. SPIE 11433, Twelfth International Conference on Machine Vision (ICMV 2019), 114331A (31 January 2020); doi: 10.1117/12.2557519

SPIE Event: Twelfth International Conference on Machine Vision, 2019, Amsterdam, Netherlands 


\title{
Automatic artery/vein classification in colour retinal images
}

\author{
Martinez-Perez, M Elena ${ }^{1,2}$; Parker, Kim H³ ${ }^{3}$ Witt, Nick ${ }^{3}$; Hughes, Alun D ${ }^{4,5}$ and Thom, Simon A M ${ }^{2}$ \\ ${ }^{1}$ Department of Computer Science, Institute of Research in Applied Mathematics and Systems, \\ National Autonomous University of Mexico, CDMX, \\ ${ }^{2}$ Faculty of Medicine, National Heart \& Lung Institute, Imperial College, London, UK, \\ ${ }^{3}$ Department of Bioengineering, Imperial College, London, UK, \\ ${ }^{4}$ Institute of Cardiovascular Science, University College London, London, UK, \\ ${ }^{5}$ MRC Unit for Lifelong Health and Ageing at UCL, London, UK.
}

\begin{abstract}
Quantitative imaging of retinal arteries and veins offers unique insights into cardiovascular and microvascular diseases but is laborious. We developed and tested a method to automatically identify arterial/venular (A/V) vessels in digital retinal images in conjunction with a semi-automatic segmentation technique. Methods of segmentation of blood vessels and the optic disc (OD) was performed as previously described, using a dataset of 10 colour fundus images. Using the $\mathrm{OD}$ as a reference a graph representation was constructed using the vessel skeletons. Vessel bifurcations and crossings were identified based on direction and local geometry, and $\mathrm{A} / \mathrm{V}$ classification was carried out by fuzzy logic classification using colour information. Results were compared with expert classification. Preliminary results showed an average true positive rate for arteries of $\mathrm{TPR}_{\mathrm{A}}=0.83$ and $\mathrm{TPR}_{\mathrm{V}}=0.74$ for veins. With an overall average of $\mathrm{TPR}_{\text {all }}=0.79$ for both vessel type jointly. Computer-based systems can assess local and global aspects of the retinal microvascular architecture, geometry and topology. Automated A/V classification will facilitate efficient cost-effective assessment of clinical images at scale.
\end{abstract}

Keywords: Automatic classification, artery/vein classification, fundus images, graph representation, fuzzy c-means.

\section{INTRODUCTION}

The retina is one place in the human body where an almost entire network of blood vessels can be viewed directly in vivo and examined for pathological changes. By using an ophthalmoscope to look through the pupil, one can see a magnified image of the retina and the blood vessels that course across its anterior surface.

Blood vessel morphology can be an important indicator for many diseases such as diabetes, hypertension and arteriosclerosis. Arteries and veins have many observable features such as diameter, length, branching angle, colour and tortuosity. The measurement of geometrical or topological changes can be applied to a variety of clinical studies: screening, diagnosis and evaluation of treatment [1-3].

The retina is perfused by the central retinal artery which enters the eye in, or beside the optic nerve and branches in the optic disc generating arterial/arteriolar trees that supply the capillaries of the retina. The retinal venules/veins collect the blood from the capillaries and merge into a single central retinal vein that exits the eye through the optic nerve. Both the arterial and venous vessels form binary trees which are relatively random in structure. It is observed that artery-artery and vein-vein crossing seldom, if ever, occur. Additionally, arteries look brighter and thinner than corresponding veins.

Many methods for artery/vein (A/V) classification have been published [4-7]. They differ mainly in the way that they use local information (colour, vessel size, etc.) and global structural/network information. In this work we report a methodology that combines structural information with graph representation and features based on colour to classify vessel trees using fuzzy c-means. The method is preliminary and has been applied and tested using a local database of 10 fundus colour images which have been semi-automatically segmented, and manually marked by one of the authors for evaluation purposes.

Twelfth International Conference on Machine Vision (ICMV 2019), edited by Wolfgang Osten, Dmitry Nikolaev, Jianhong Zhou, Proc. of SPIE Vol. 11433, 114331A · C 2020 SPIE CCC code: $0277-786 \mathrm{X} / 20 / \$ 21 \cdot$ doi: $10.1117 / 12.2557519$ 


\section{METHOD}

The method involves three main tasks: i) segmenting blood vessels and the optic disc (OD), erasing the optic disc area from the binary image of vessels; separating the resultant binary image into connected objects (forests); for every forest in the image: thinning the segmented blood vessels to produce their skeletons and detecting terminals and branching points in the skeleton; ii) correcting the crossing points in the skeleton by generating a graph representation of the network; and extracting the vessel trees; iii) computing the fuzzy c-means of the colour features of each forest in a cascade fashion obtaining two classes, $\mathrm{P}$ and $\mathrm{Q}$, until all forest are classified. Finally vessel trees are classified as A/V from classes $\mathrm{P}$ and $\mathrm{Q}$ based on the values of the average cluster centres. Figure 1 depicts a diagram of the method.

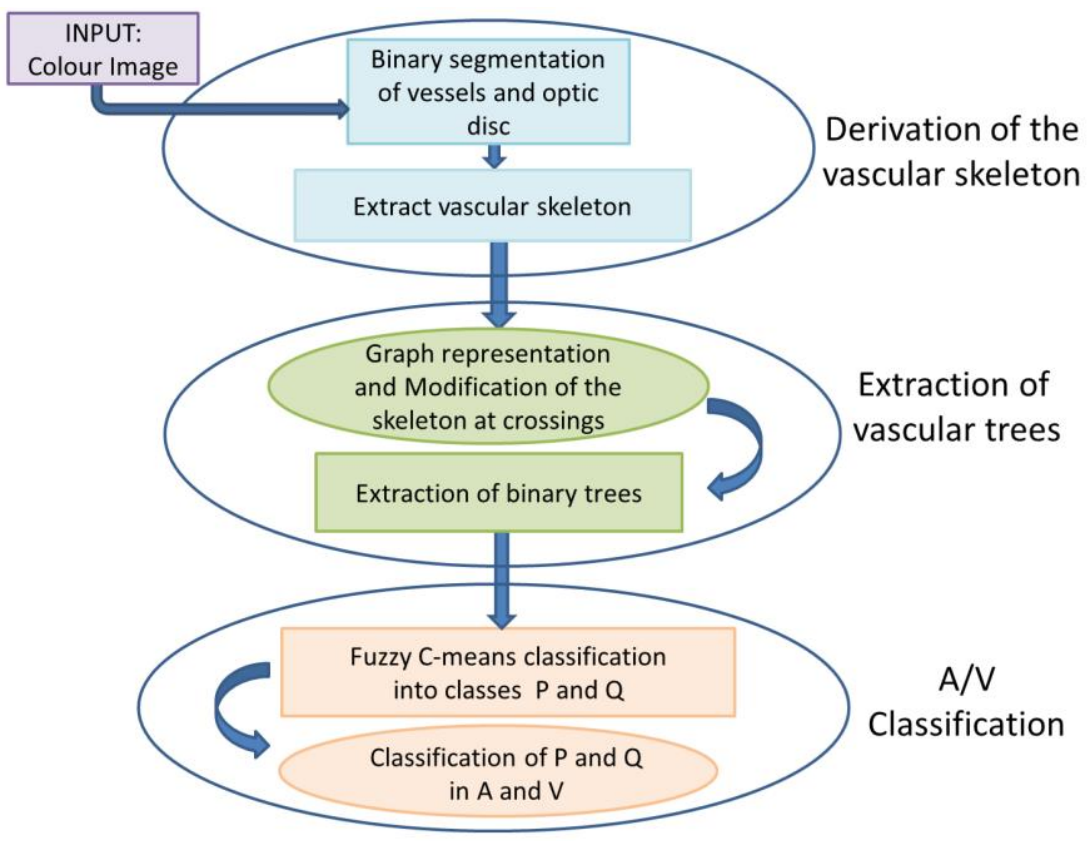

Figure 1. Diagram of the stages for the A/V classification method.

The input image for the method is the colour image, the segmented blood vessels and the localisation of the OD, which are derived from the colour image, as shown in Figure 2.

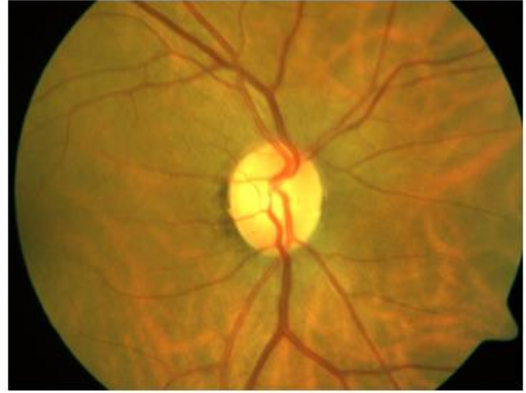

(a)

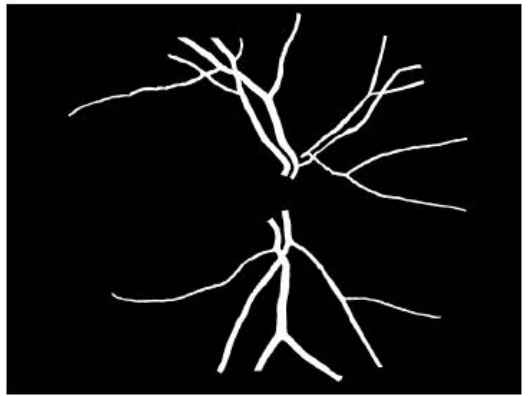

(b)

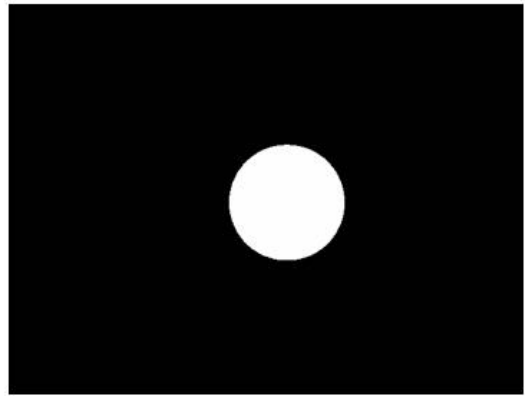

(c)

Figure 2. a) Colour image, b) segmented blood vessels and c) localisation of the optic disc. 


\subsection{Derivation of the vascular skeletons}

\subsubsection{Binary segmentation of vessels and OD}

Blood vessels are segmented and the centre of the OD is localised and used as a reference to establish binary rooted trees $[8,9]$. For each image, a connected forest with one, two or more rooted trees, are labelled and analysed independently (Figure 3(a)).

\subsubsection{Extraction of vascular skeletons}

For each connected forest, the skeletons of the vascular trees are obtained by a thinning process where pixels are eliminated from the boundaries towards the centre without destroying connectivity [10]. From the skeleton lines, terminal and branching points are identified and marked. Pixels with only one neighbour in a $3 \times 3$ neighbourhood in the skeleton are labelled as terminal points, and pixels with 3 neighbours are labelled as bifurcation points. Bifurcation points are labelled as $-r$, where $r$ is the radius of the maximum circle centred on that point that fits inside the boundary of the segmented vessel, see Figure 3(b). Three different configurations of circles exist: single, two intersecting and three intersecting circles. Some of these configurations will be used during the task of correction of crossing points.

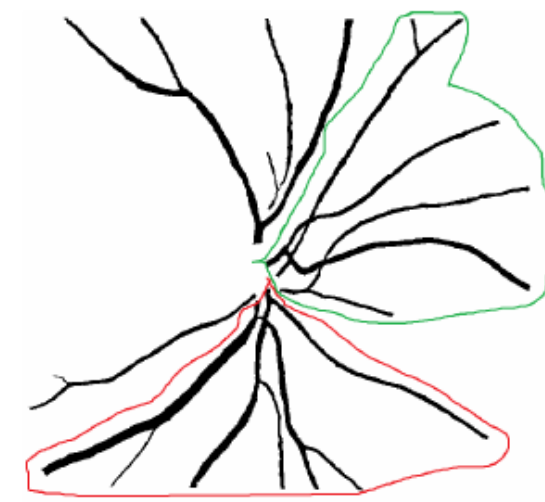

(a)

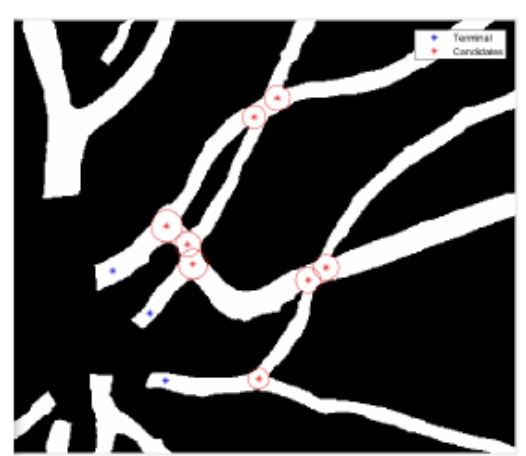

(b)

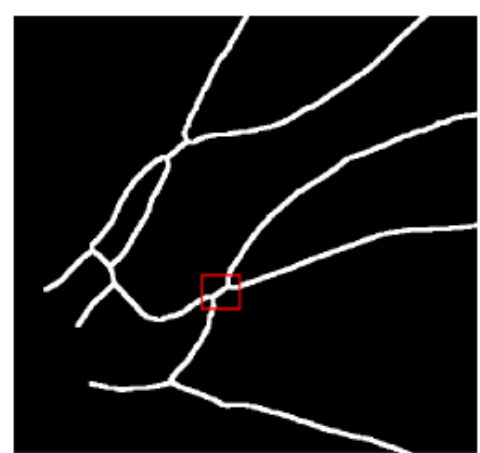

(c)

Figure 3. Identification of terminal, branching and crossing points. a) Binary image is separated into connected objects (forests), with roots. One, two, three or more rooted trees in each forest, b) Using the skeleton, terminal and branching points are identified and circles are marked at bifurcations as: single circle, two, or three intersecting circles. c) At first, only two intersecting circles are reconised as crossings, the skeleton is corrected and marked as a crossing, using a variable size window, the bounding box that includes the area of both circles.

\subsection{Extraction of vascular trees}

\subsubsection{Graph representation}

Using the segments of the skeletons as edges and the terminal and bifurcating points as nodes, a graph is built (Figure 4(a)). A connectivity matrix is defined which contains the connectivity information between nodes. The degree of a node (number of edges connected to it) indicates its topology: $1=$ terminal; $3=$ bifurcation; $4=$ crossing (Figure 4(b)). 


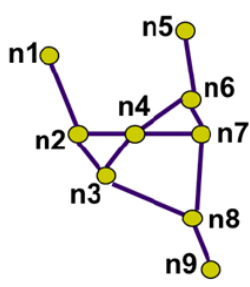

(a)

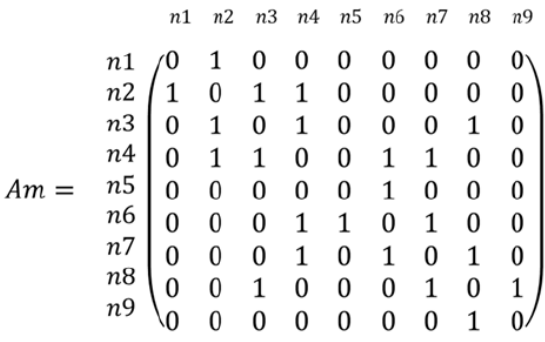

(b)

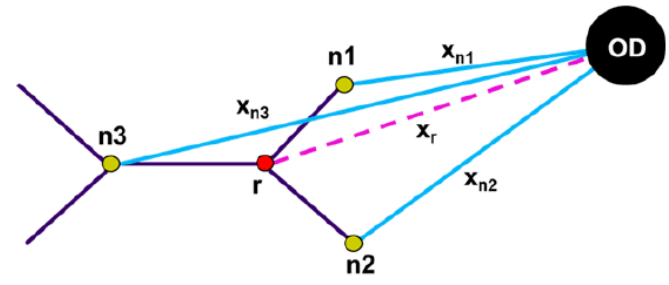

(c)

Figure 4. Graph representation and identification of crossing points. a) Vessel segments are edges and terminal or branching points are nodes. b) Adjacency matrix between nodes, the sum of the elementes per row identifies the degree of the node. c) Identifying a crossing point, where OD represents the optic disc, $r$ is the node being tested as "proper/improper", $n_{i}$ are the adjacent nodes to $r$, and $x_{i}$ are the respective distances from the nodes to the centre of the OD.

\subsubsection{Modification of skeleton at crossing points}

Using only the two intersecting circles identified during the task described in section 2.1.2 (Figure 3(b)), a window of a variable size is defined as the bounding box around its area (Figure 3(c)); if the skeleton intersects the window at four points, this node is defined as a crossing point and a node of degree 4 is updated in the graph. This process fails to identify crossings when two vessels cross at a very acute angle so that the two circles do not intersect, given adjacent single circles. Therefore these single circles are not always "proper" bifurcation points and can give rise to errors in node classification.

Since the rooted trees leave the OD and branch in the direction of the periphery of the retina, a "proper" bifurcation will diverge in a distal rather than a proximal direction. Therefore, we used the center of the OD as a reference location and for every node of degree 3 , we check if it is branching away from the OD (a "proper" node) or not (an "improper" node) (Figure 4(c)). For each node $r$ we calculate the distances of the three connected nodes from the OD.

$$
d_{i}=x_{n i}-x_{r}
$$

If two, out of three, $d_{i}$ are negative then $r$ is an "improper" node and should be joined with the node with a positive $d_{i}$. The skeleton and the adjacency matrix are updated accordingly. Figure 5(a) shows the final version of the skeleton marked as a forest with three rooted trees, where terminals are marked in blue, branches in red and crossings points in green.

\subsubsection{Extraction of binary trees}

Finally, we remove the nodes of degree 4 from the graph and update the adjacency matrix accordingly to separate the rooted tree graphs, see Figure 5(b). 


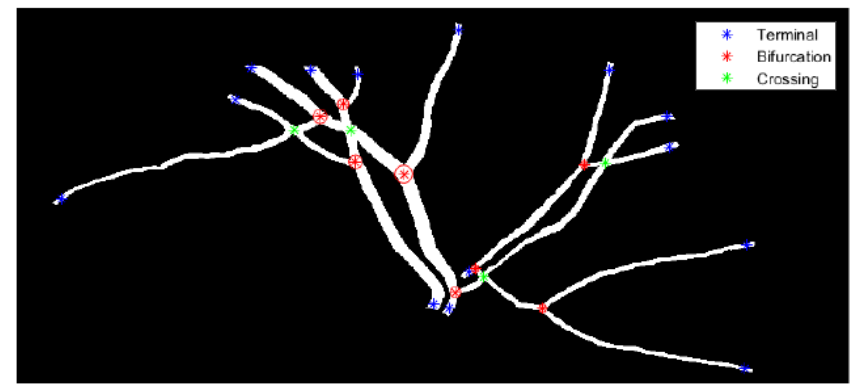

(a)

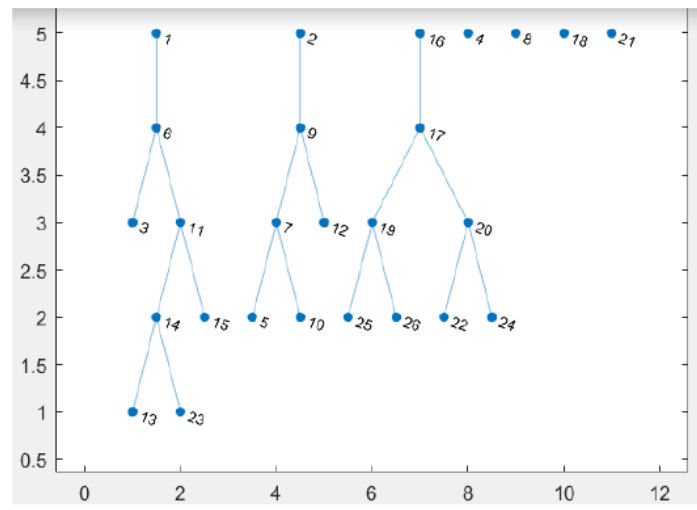

(b)

Figure 5. Forests with three rooted trees. a) Final tree skeleton marked based on the information of the graph, terminal points in blue, branching points in red and crossing point in green, and b) by eliminating the nodes of degree 4 , the binary trees can be extracted.

\section{$2.3 \quad \mathrm{~A} / \mathrm{V}$ classification}

The classification process runs in the following fashion: first all two rooted forests are classified into two classes $\mathrm{P}$ or $\mathrm{Q}$, using fuzzy c-means of a local feature vector. Since we know a priori that arteries only cross veins, the two trees must be of different classes. Then three or more rooted forests are classified knowing that a tree can only cross a tree of a different class to itself. All cluster centers obtained by every forest classification are averaged to get a unique cluster centre per class $\mathrm{P}$ and $\mathrm{Q}$. Finally all single rooted trees are classified depending on the distance of the mean of their feature vector to that of the nearest average cluster centre.

Some important features from the colour fundus images are taken into account when defining the feature vector: first, as the wavelength of the green light is largely absorbed by blood but is partially reflected by the retinal pigmentation, green light provides excellent contrast and the best overall view of the fundus and the retinal vasculature. Second, in red light, retinal vessels look lighter and are less obvious, with the arteries containing oxygenated haemoglobin appearing lighter compared to the veins which contain $\sim 40 \%$ de-oxygenated haemoglobin [11]. Blue light provides limited additional information to red and green. The fundus images are acquired in RGB colour mode and the feature vector used herein is composed of the red $(\mathrm{R})$ and the green $(\mathrm{G})$ channels.

\subsubsection{Fuzzy c-means classification into classes $P$ and $Q$}

The information in the $\mathrm{R}$ and $\mathrm{G}$ channels is extracted for all of the pixels along the skeleton line of the root and first branch vessels (RFB) for each root in the connected forest. The Fuzzy c-means technique is based on the minimization of the objective function:

$$
J_{m}=\sum_{i=1}^{P} \sum_{j=1}^{N} \mu_{i j}^{m}\left\|v_{i}-c_{j}\right\|^{2}
$$

where $P$ is \# of data points. $N$ is the \# of clusters. $m$ controls the degree of fuzzy overlap. $v_{i}$ is the $i$ th data point. $c_{j}$ is the centre of the $j$ th cluster. $\mu_{i j}$ is the degree of membership of $v_{j}$ in the $j$ th cluster. Figure $6(\mathrm{a})$ shows the plot of $\mathrm{G}$ mean value versus R mean value, with pixel membership of classes P (magenta) and Q (green) illustrated, for $m=8$. 


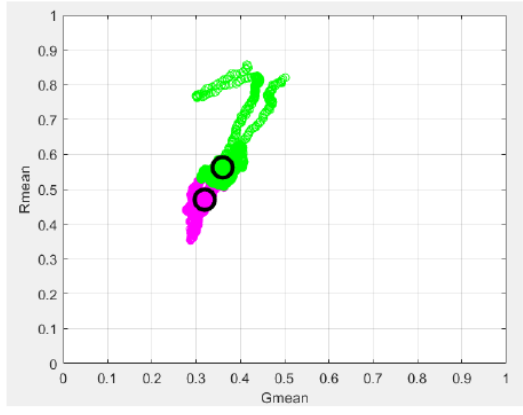

(a)

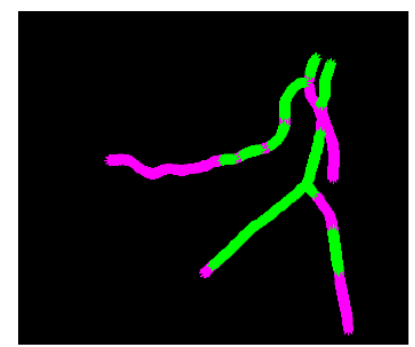

(b)

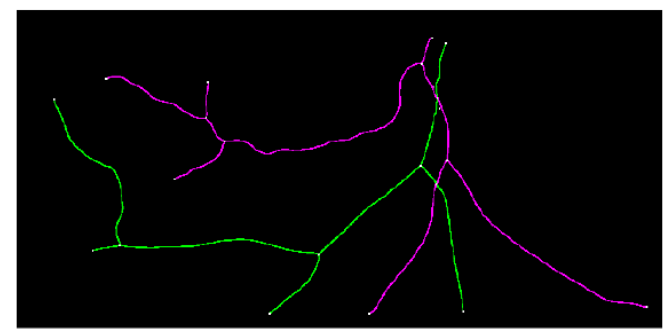

(c)

Figure 6. Fuzzy c-means of a two rooted forest. a) Cluster data with cluster centres, features used: green ( $x$-axis) and red ( $y$-axis) channels, for $m=8$; b) pixels membership for each class $\mathrm{P}$ or Q using only the pixels of the skeleton that belong to the root and first branches of each tree, and c) final classification of trees based on the ratio of $\mathrm{P}$ and $\mathrm{Q}$ for each tree.

For each two rooted connected forest the membership of classes $\mathrm{P}$ and $\mathrm{Q}$ is computed. Figure 6(b) shows the respective membership class for every pixel of the RFB section of the rooted trees. As it can be seen from Figure 6(b), each RFB section can have pixels that belong to one class or the other, but we know that both trees should be of different class (since they cross). To classify each RFB section of tree as belonging to a single class, a proportion for individual bifurcation is computed as follows:

$$
\begin{aligned}
& N_{i}=P_{i}+Q_{i} \\
& T_{i}=\frac{P_{i}}{N_{i}} \\
& \text { Class } P=\max _{i} T_{i} \\
& \text { otherwise Class } Q
\end{aligned}
$$

where $i=\{1,2\}, N_{i}=$ the number of pixels classified as $P$ plus number of pixels classified as $Q$ for the RFB section of the tree $i ; T_{i}=$ proportion of pixels of class $\mathrm{P}$ in the RFB section of the tree $i$; the maximum proportion between two RFB section of trees is assigned as class $\mathrm{P}$ and otherwise as class Q. Figure 6 (c ) shows the final P/Q classification for both complete trees after applying the proportion rule (Eq. 3) and extending the RFB classification to the rest of the tree.

After classifying all connected forests of two rooted trees, a similar idea is applied to three or more rooted trees as long as the classification is made with pairs of intersecting trees. An average of all cluster centers is computed. Finally all single rooted trees are classified depending on the distance of the mean of their feature vector to that of the nearest average cluster centres.

\subsubsection{Classification of $P$ and $Q$ into $A$ and $V$}

The $\mathrm{P}$ and $\mathrm{Q}$ classifications are based only on relative distances within the feature vectors in the image; No prior knowledge is used in the P/Q classification. Assigning P and Q to A (arteries) and V (veins) is done at the very last step of the process. Using prior knowledge that veins will have a lower intensity than arteries, the class cluster nearest to the origin of the $\mathrm{G}$ mean vs. R mean plot is assigned to be $\mathrm{V}$, and the class cluster furthest from the origin is assigned to be A.

\section{RESULTS}

Figure 7 shows the results using a database of 10 retinal colour images. The first column of Figure 7 corresponds to the input colour image, the second classification into classes P (magenta) and Q (green), the third to the classification into classes A (red) and V (blue); and the last column represents the manual classification made by one of the authors, where 
$\mathrm{A}=$ red and $\mathrm{V}=\mathrm{blue}$. The rows of Figure 7 from top to bottom correspond to the best, medium and worst performance in the 10 images.
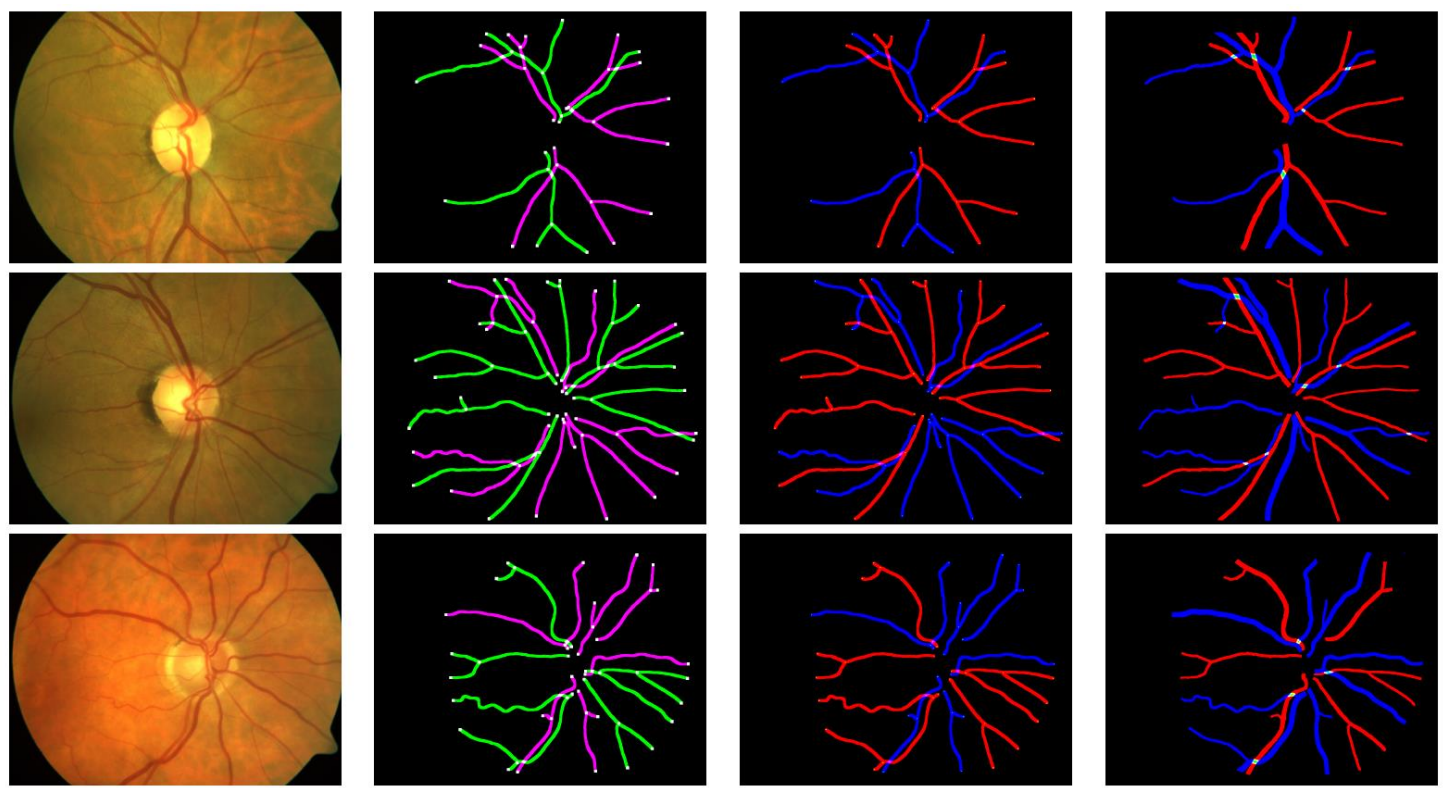

Figure 7. Examples of $\mathrm{A} / \mathrm{V}$ classification results. First column: colour image; second column: classes $\mathrm{P}$ and $\mathrm{Q}$; third column: classes $\mathrm{A}$ and $\mathrm{V}$ using the algorithm; and final column: the hand-classified $\mathrm{A}$ and V. From top to bottom: best classification performance TPR ${ }_{\mathrm{A}}=0.92$ (Image 2 from Table 1); $\mathrm{TPR}_{\mathrm{V}}=0.92 ;$ medium $\mathrm{TPR}_{\mathrm{A}}=0.82, \mathrm{TPR}_{\mathrm{V}}=0.81($ Image 8 from Table 1$) ;$ and worst $\mathrm{TPR}_{\mathrm{A}}=0.66 ; \mathrm{TPR}_{\mathrm{V}}=0.56($ Image 4 from Table 1$)$.

Performance was measured by computing the true positive rate for arteries and veins compared to that of the manual classification, which was considered ground truth (GT). For each centerline pixel from the complete trees, the true positive rate for arteries $\left(\mathrm{TPR}_{\mathrm{A}}\right)$ is defined as the ratio of correctly classified arterial pixels to all centerline pixels labelled as artery in the GT. The true positive rate for veinular $\left(\mathrm{TPR}_{\mathrm{V}}\right)$ is defined as the ratio of correctly classified vein pixels to all centerline pixels labelled as vein in the GT. The overall $\mathrm{TPR}_{\text {all }}$ is the ratio of all centerline pixels correctly classified (arteries and veins) to all centerline pixels labelled as artery or vein in GT. Table 1, summarises the results for each of the 10 images tested.

Table 1. True positive rate for arteries $\left(\mathrm{TPR}_{\mathrm{A}}\right)$, for veins $\left(\mathrm{TPR}_{\mathrm{V}}\right)$ and the overall values for both $\left(\mathrm{TPR}_{\mathrm{all}}\right)$. The values in bold correspond to the images in Figure 7.

\begin{tabular}{|c|c|c|c|}
\hline Image & TPRA & TPRV & TPRall \\
\hline 1 & 0.80 & 0.35 & 0.62 \\
\hline $\mathbf{2}$ & $\mathbf{0 . 9 2}$ & $\mathbf{0 . 9 2}$ & $\mathbf{0 . 9 2}$ \\
\hline 3 & 0.76 & 0.94 & 0.85 \\
\hline $\mathbf{4}$ & $\mathbf{0 . 6 6}$ & $\mathbf{0 . 5 6}$ & $\mathbf{0 . 6 1}$ \\
\hline 5 & 0.93 & 0.63 & 0.79 \\
\hline 6 & 0.96 & 0.53 & 0.75 \\
\hline 7 & 0.71 & 0.93 & 0.81 \\
\hline $\mathbf{8}$ & $\mathbf{0 . 8 2}$ & $\mathbf{0 . 8 1}$ & $\mathbf{0 . 8 2}$ \\
\hline 9 & 0.80 & 0.93 & 0.86 \\
\hline 10 & 0.93 & 0.82 & 0.88 \\
\hline Average & 0.83 & 0.74 & 0.79 \\
\hline
\end{tabular}




\section{CONCLUSIONS}

We have presented an automatic A/V classification method based on global analysis of the vessel network together with the local colour information, using a fuzzy c-means classification from already extracted binary tree graphs, integrating the properties of connectivity as well as the physiological characteristic that arteries and veins ramify in a distal direction from the OD. Preliminary results showed an average of true positive rates $\mathrm{TPR}_{\mathrm{A}}=0.83$ for arteries and $\mathrm{TPR}_{\mathrm{V}}=0.74$ for veins. With an overall average of $\mathrm{TPR}_{\mathrm{all}}=0.79$ for both vessel types jointly. The main problem accounting for suboptimal values for TPR is the classification of single rooted trees, as seen in the second and third rows of Figure 7. Other authors have reported different metrics for evaluation, they have used different databases as well as different GTs [4-6]. Thus, the comparison of our results with others is not a straight forward task. Future work will be focused on improving the single rooted tree classification, testing the algorithm performance with a local larger image database as well as on public databases for comparisons. Computer-based systems can assess local and global aspects of the retinal microvascular architecture, geometry and topology. Automated A/V classification will facilitate efficient cost-effective assessment of clinical images at scale.

\section{REFERENCES}

[1] Struijker, HAJ, le Noble, JLM, Messing, MWJ, Huijberts, MSP, le Noble, FAC, and Van Essen, H. "The microcirculation and hypertension," J. Hyperten., vol. 10, pp. S147-S156, 1992.

[2] Stanton, AV, Wasan, B, Cerutti, A, Ford, Marsh, SR, Sever, PS, Thom,SAM, and Hughes, AD. "Vascular network changes in the retina with age and hypertension," J. Hyperten., vol. 13, pp. 1724-1728, 1995.

[3] King, LA, Stanton, SV, Sever, PS, Thom, SAM, and Hughes, AD. "Arteriolar length-diameter (1:d) ratio: A geometric parameter of the retinal vasculature diagnostic of hypertension," J. Hum. Hypertens., pp. 417-418, 1996..

[4] Huang, F., Dashtbozorg, B. \& Romeny, B.M..H. "Artery/vein classification using reflection features in retina fundus images". Machine Vision and Applications (2018) 29: 23.

[5] Xiayu Xu, Wenxiang Ding, Michael D. Abràmoff, Ruofan Cao. "An improved arteriovenous classification method for the early diagnostics of various diseases in retinal image".Computer Methods and Programs in Biomedicine, Vol. 141, 2017, Pages 3-9.

[6] Favali, M., Abbasi-Sureshjani, S., ter Haar Romeny, B. et al. "Analysis of vessel connectivities in retinal images by cortically inspired spectral clustering". J Math Imaging Vis (2016) 56: 158.

[7] Hadi Hamad, Domenico Tegolo and Cesare Valenti. "Automatic detection and classification of retinal vascular lanmarks". Image Analysis \& Stereology. 33(3), 189-200. 2014.

[8] Martinez-Perez, ME, Hughes, AD, Thom, SAM, Bharath, AA and Parker, KH. "Segmentation of blood vessels from red-free and fluorescein retinal images". Medical Image Analysis. 11 (1): 47-61, 2007.

[9] Martinez-Perez ME, Witt N, Parker KH, Hughes AD, Thom SAM. 2019. "Automatic optic disc detection in colour fundus images by means of multispectral analysis and information content". PeerJ 7:e7119 https://doi.org/10.7717/peerj.7119

[10] Lam, L., Seong-Whan Lee, and Ching Y. Suen, "Thinning methodologies - a comprehensive survey," IEEE Transactions on Pattern Analysis and Machine Intelligence, Vol 14, No. 9, September 1992, page 879.

[11] Bennett TJ. 2011. "Monochromatic fundus photography". Ophthalmic photographer's society. Available at: https://www.opsweb.org/page/monochromatic (accessed December 2018).

\section{ACKNOWLEDGMENTS}

Research supported by CONACYT, PASPA-DGAPA from UNAM, and the NIHR Imperial BRC. AH receives support from the British Heart Foundation (CS/13/1/30327, PG/13/6/29934, PG/15/75/31748, CS/15/6/31468, PG/17/90/33415, IG/18/5/33958), the National Institute for Health Research University College London Hospitals Biomedical Research Centre, the UK Medical Research Council (MR/P023444/1) and works in a unit that receives support from the UK Medical Research Council (MC_UU_12019/1). 